\title{
Estímulo à prática multidisciplinar no ensino de Computação e Design através de um evento de programação focado em problemas
}

\author{
Daniela Brauner ${ }^{1}$, Pablo Margreff ${ }^{2}$, Tatiana Tavares ${ }^{2}$, \\ Vinicius Kruger da Costa ${ }^{3}$, Alexandre L. Silva ${ }^{4}$ \\ ${ }^{1}$ Escola de Administração - Universidade Federal do Rio Grande do Sul (UFRGS) \\ Porto Alegre - RS - Brasil. \\ ${ }^{2}$ CDTec - Centro de Desenvolvimento Tecnológico - Universidade Federal de Pelotas \\ (UFPel) \\ Pelotas - RS - Brasil. \\ ${ }^{3}$ Coordenadoria de Design - Instituto Federal de Educação, Ciência e Tecnologia \\ Sul-rio-grandense (IFSul) \\ Pelotas - RS - Brasil. \\ ${ }^{4}$ Conrad Caine - Possible \\ Pelotas - RS - Brasil. \\ ${ }^{1}$ daniela.brauner@ufrgs.br, ${ }^{2}$ \{pmargreff, tati... $\}$ @inf.ufpel.edu.br,
viniciusdacosta@pelotas.ifsul.edu.br, ${ }^{4}$ alexandre.silva@possible.com
}

Abstract. Hackathons are programming events focused on problem solving through a collaborative and competitive dynamics. The informal nature of hackathons make them excellent platforms for learning, able to attract diverse audience, especially those who would like to try something different from the traditional model of the classroom. This paper discusses an experience of a hackathon with students from Computing and Design. The experiment was held in an IT company and it has university and industry mentors. Despite the competitive nature, the hackathon provide an important stimulus tool for learning, collaborative and multidisciplinary work.

Resumo. Hackathons são eventos de programação focados na resolução de problemas através de uma dinâmica colaborativa e competitiva. A natureza informal dos hackathons o tornam excelentes plataformas de aprendizagem, capazes de atrair público diversificado, especialmente, àqueles que gostariam de experimentar algo diferente do dia-a-dia de sala de aula. Neste artigo é discutido um relato de experiência de um hackathon com estudantes de Computação e Design. A experiência realizada dentro de uma empresa contou com mentores da universidade e indústria. Apesar da natureza competitiva, o hackathon se mostrou uma importante ferramenta de estímulo ao aprendizado e ao trabalho colaborativo e realmente multidisciplinar. 


\section{Introdução}

Segundo Heikki Topi e Allen Tucker (2014) um hackathon é um evento de programação em Computação focado em problemas. Briscoe e Mulligan (2014) acrescentam que hackathons são instrumentos importantes para encorajar a experimentação e criatividade, aspectos essenciais à inovação. Dessa forma, o hackathon pode ser visto como uma ação conjunta entre programadores e outros stakeholders $^{1}$ que atuam colaborativamente no desenvolvimento de software engajados de forma intensa e desafiadora durante um curto período de tempo.

Com o intuito de propor esse desafio para a comunidade da parte sul do Rio Grande do Sul, em novembro de 2015 a Universidade Federal de Pelotas (UFPel) e o Instituto Federal Sul-Riograndense (IFSul) organizaram e executaram o CodeArena. O CodeArena oportunizou uma experiência multidisciplinar voltada para problemas práticos através de um evento no estilo hackathon. O objetivo deste evento foi oferecer para alunos dos cursos de Computação e de Design, a interação entre áreas, que é pouco abordada durante a graduação. Considerando que atualmente a interdisciplinaridade é praticamente um requisito para o trabalho em TI, o CodeArena ofereceu essa oportunidade para a troca de experiência entre alunos de diferentes instituições de ensino, fazendo com que a troca bilateral de diferentes olhares contribuísse para motivar os alunos de ambas as instituições envolvidas.

Este artigo descreve a concepção e execução do hackathon CodeArena no intuito de motivar a organização de práticas multidisciplinares como estímulo ao ensino e aprendizado em Computação e Design. Para tanto, primeiramente, é apresentada uma visão geral da dinâmica dos hackathons destacando os principais pontos para organizar um evento com essas caracteríticas. E, então, é discutida a abordagem utilizada no hackathon CodeArena através do detalhamento das atividades realizadas. Por fim, são abordadas as as lições aprendidas com a realização de um evento deste tipo, bem como as vantagens para a universidade e as empresas, nas diferentes perspectivas de envolvimento.

\section{A Dinâmica dos Hackathons}

A origem da palavra hackathon é a combinação de hack e marathon. A palavra "hack" remete ao senso de investigação e exploração inerente à atividade de programação. Já a palavra "marathon" está relacionada a modalidade esportiva maratona que é uma corrida ininterrupta de longa distância que geralmente ocorre nas ruas, desafio, exaustão e competição são aspectos intrínsecos da maratona. Dessa forma, um hackathon envolve a investigação de soluções que envolvam Computação até a exaustão através de uma competição.

Briscoe e Mulligan (2014) destacam que hackathon é uma atividade que pode possuir muitas versões diferentes e, como tal, não se limita a uma linha particular. No entanto, existem alguns elementos que se destacam como "universalmente necessários", como é o caso da colaboração. Embora seja difícil estabelecer um conjunto de melhores

\footnotetext{
${ }^{1}$ Stakeholder é o termo utilizado para qualquer pessoa ou entidade envolvida com o processo de software, por exemplo, designers, testadores, cliente.
}

Proceedings of the WEI'2016 (Julho 2016) 
práticas, os autores abordam princípios gerais que podem/devem ser observados: (i) Hackathons são orientados a produção de saídas; (ii) Hackathons devem ser inclusivos; (iii) Hackathons devem oportunizar o aprendizado e o compartilhamento de conhecimentos; (iv) Hackathons devem incentivar o aprendizado através da tentativa-erro; (v) Hackathons necessitam de conectividade; (vi) Hackathons são beneficiados por um local inspirador, acolhedor e confortável; (vii) Hackathons são atividades colaborativas e em grupos. Líderes dos grupos devem ser incentivados, mas não devem ser obrigatórios.

A Fig.1 apresenta uma visão da dinâmica dos hackathons baseada na proposta da Clever Together ${ }^{2}$ para uma ação chamada \#ChangeChallenge. Nessa visão observados os pontos essenciais para a realização de um hackathon: (i) o entendimento do problema através da compreensão do seu contexto através da delimitação das suas fronteiras; (ii) a pesquisa de soluções relacionadas que possam agregar na proposta a ser desenvolvida; (iii) o estabelecimento de prioridades para identificar atividades e entregáveis o que compõe o planejamento do grupo; (iv) a etapa de "hands-on", isto é, a realização das atividades planejadas para o desenvolvimento da solução.

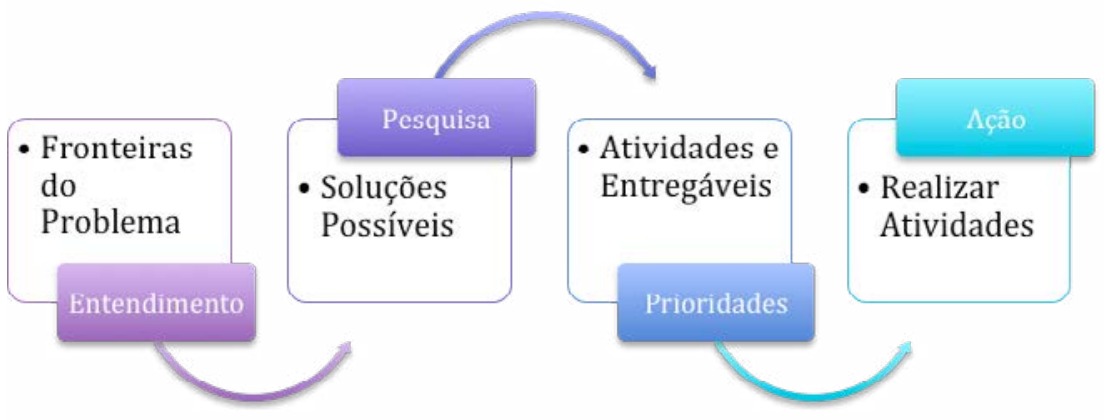

Figura 1. Visão geral da metodologia estilo hackathon.

De forma complementar a dinâmica apresentada por Briscoe e Mulligan (2014) destaca que normalmente os hackathons começam com uma ou mais apresentações sobre o evento, incluindo a apresentação do formato geral do evento, seus desafios e os prêmios, se disponível. O desafio é parte primordial da dinâmica do hackathon, na qual esta decisão pode ser feita em conjunto com os participantes ou de forma orientada pela organização.

A formação das equipes pode considerar interesses individuais e habilidades. Uma vez formada a equipe, é a hora da "largada" quando o trabalho principal do hackathon começa, que pode durar de algumas horas a vários dias. Nesse caso, se alimentar e dormir são atividades que ocorrem geralmente no local do evento. Ao final, cada grupo apresenta seus resultados que muitas vezes envolvem protótipos de software. Nesse momento, uma banca de juízes seleciona as equipes vencedoras e, então, os prêmios são entregues. Em muitos hackathons, os juízes são os próprios organizadores, bem como os patrocinadores do evento.

Segundo Fassbinder et al. (2012) o desenvolvimento de competições de conhecimento é uma forma de estimular os alunos a aprimorarem seus conhecimentos

\footnotetext{
${ }^{2} \mathrm{http}: / / \mathrm{clevertogether} . c o m / p u b l i c /$ healthcare/change-challenge-methodology/

Proceedings of the WEI'2016 (Julho 2016)
} 
em determinada área. Os autores acrescentam que ao ingressarem numa disputa os participantes se vêem engajados na missão de provar o seu valor e o seu conhecimento. Além disso, guiados pelo instinto competitivo, não medem esforços para buscar ainda mais conhecimento e transpor qualquer barreira que surja em seu caminho. Adicionalmente, Nandi e Mandernach (2016) destacam a importância do aspecto informal de aprendizado inerente aos hackathons. Dessa forma, a dinâmica dos hackathons pode estimular a experiência multidisciplinar e colaborativa para a resolução de problemas, especialmente, envolvendo a programação de soluções. A competição e a imersão inerentes ao formato do evento impulsionam a busca por soluções possíveis para os desafios propostos.

\section{Atividades Realizadas}

A primeira etapa foi a chamada de ideias, para tanto a organização definiu um período de um mês para que qualquer pessoa, sendo parte ou não da comunidade acadêmica, submetesse um problema e sua respectiva sugestão de solução através do site $^{3}$ do evento como ilustrado na Fig.2 (a). No total foram submetidas 19 problemas, destes foram escolhidos 6 para o desenvolvimento durante o evento, por uma comissão formada por pesquisadores da universidade, através de uma análise que contemplou os seguintes critérios: originalidade, viabilidade do produto, viabilidade técnica e impacto social.

A formação das equipes (times) foi realizada em duas etapas online: cadastramento dos participantes e candidatura do participantes para as ideias selecionadas conforme ilustrado na Fig. 2 (b). Cada time deveria agrupar 3 participantes, sendo 2 (duas) vagas para a área de Computação e 1 (uma) para a área de Design. Nesta primeira edição do CodeArena foram oferecidas 24 vagas no total, sendo preenchidas, destas, 19 vagas. Quanto à formação, 5 alunos eram do Design e 14 da Computação sendo, quanto ao gênero, apenas 3 pessoas do sexo feminino e 16 do sexo masculino.

Um resultado que já podemos destacar foi o envolvimento de diferentes cursos e instituições nesta etapa de formação das equipes. Dentre os cursos da área da Computação foram envolvidos os cursos de: Bacharelado em Ciência da Computação (UFPel), Bacharelado em Engenharia de Computação (UFPel), Pós Graduação em Computação (UFPel), Técnico em Eletrônica (IFSul), Licenciatura para Computação (IFSul) e Tecnólogo em Sistemas para Internet (IFSul). Já para os cursos da área de Design destacamos: Bacharelado em Design (IFSul), Bacharelado em Design Gráfico (UFPel) e Bacharelado em Design Digital (UFPel). Dessa forma, observamos o engajamento de alunos de nove cursos: 6 da área de Computação e 3 de Design.

Uma vez formadas as equipes pela página Web foi dado o início das atividades efetivas do hackathon. Para proporcionar o primeiro encontro dos times realizamos uma reunião na noite anterior que antecedeu o início do hackathon propriamente dito. Nessa reunião de aquecimento as equipes tiveram o primeiro contato e puderam interagir com os membros da organização. Também foram detalhadas as propostas para todos os times permitindo que todos soubessem as ideias e também para possibilitar a troca de participantes entre os times, caso houvessem interessados. Na mesma ocasião, as equipes tiveram a oportunidade de alinharem as funções dos membros dos times e

\footnotetext{
${ }^{3} \mathrm{http}: / /$ computacao.cc/codearena/

Proceedings of the WEI'2016 (Julho 2016)
} 
projetar um MVP (Minimal Viable Product) com as funcionalidades básicas que cada projeto precisaria contemplar. Esse também foi o primeiro contato com os mentores que puderam auxiliar os grupos a definir quais seriam as prioridades para o produto.

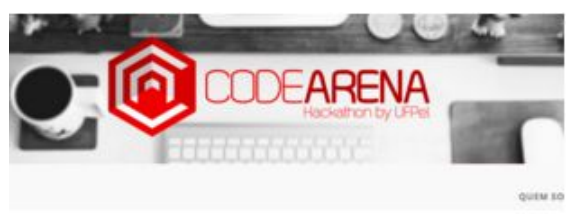

Sugestão de problema/tema

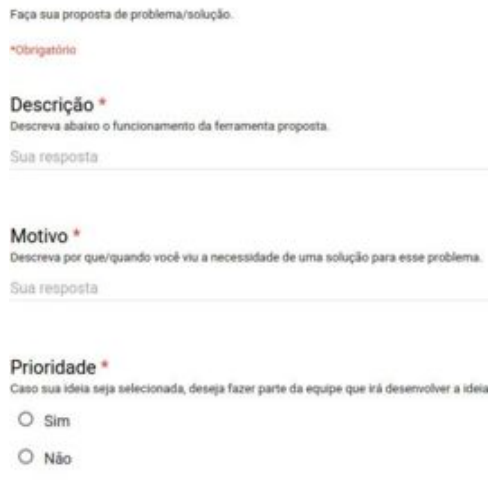

(a)
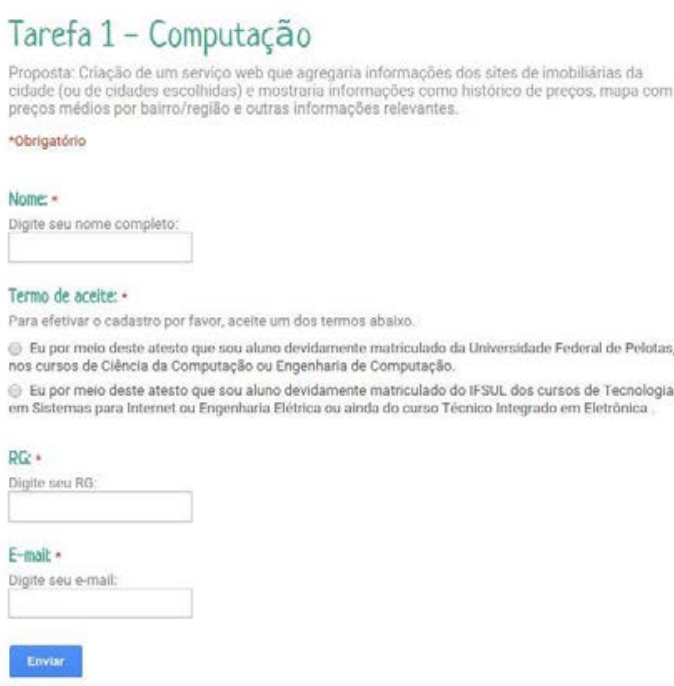

(b)

Figura 2. Em (a) screenshot da página de submissão de ideias e em (b) screenshots da página de candidaturas dos participantesdo CodeArena.

O hackathon CodeArena iniciou efetivamente suas atividades na empresa Conrad-Caine no sábado às 08:30 com a etapa de "hands-on" onde os participantes deram continuidade ao desenvolvimento dos projetos esboçados na noite anterior. Depois de ter um sketch dos projetos em mãos e de suas funcionalidades básicas, os participantes deram início ao ciclo de 36 horas de desenvolvimento dos projetos. Um observação interessante foi a integração das equipes, onde cada uma ficou livre para definir o seu processo de desenvolvimento. A Fig. 3 mostra uma das estratégias adotadas por uma das equipes que descreveu sua dinâmica através de uma quadro de kanban. As responsabilidades foram identificadas pelo perfil: design e computação (através de backend e frontend) conforme Fig.3 (a) e as atividades detalhadas em post-its (vide Fig.3(b)), bem como seu andamento na Fig.3 (c). Pela imagem é possível notar que o grupo implementou, através de cores, a separação dos papéis dos integrantes dos times.

A dinâmica dessas 36 horas de maratona, além das atividades inerentes a programação e ao design dos projetos, envolveu também atividades de entretenimento, bem como espaços para alimentação e integração entre as equipes. Jogos, desafios (vide Fig.4 (a), instrumentos musicais, pizza, lanche, bombons, salada de frutas, águas e café ficaram constantemente a disposição dos participantes. Além disso, os participantes poderiam levar colchonetes e uma sala foi disponibilizada para o seu descanso, caso necessário. Os participantes também podiam deixar o local de evento para descansar no momento em que desejassem e combinassem com sua respectiva equipe. Também ao longo do final de semana foram feitas diversas atividades, relacionadas ou não com as 
áreas envolvidas no desenvolvimento do produto, para que os participantes tivessem a oportunidade de se integrarem com os outros grupos e ter um momento mais descontraído durante o evento, além de de render alguns brindes aos seus vencedores.

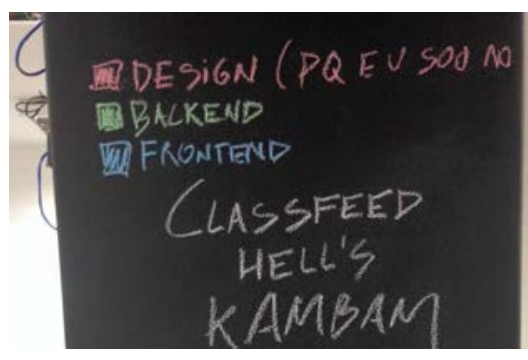

(a)

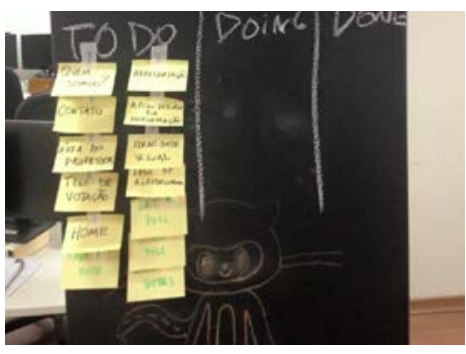

(b)

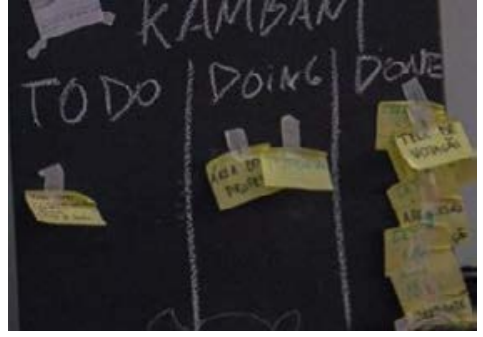

(c)

Figura 3. Quadro Kanban desenvolvido por uma das equipes. Em (a) podemos observar a divisão integrada das responsabilidades (design e computação), em (b) o detalhamento das atividades e em (c) o andamento.

As apresentações dos produtos foram divididas em duas partes, a primeira foi o pitch onde cada equipe teve 5 minutos para apresentar sua solução para os jurados e as outras equipes conforme ilustrado na Fig.4 (b). A segunda parte foi o teste dos protótipos dos projetos conforme Fig.4. (c). Durante os testes os mentores visitaram todas as equipes e utilizaram os protótipos, tiraram eventuais dúvidas e discutiram possíveis melhorias a serem implementadas em cada projeto. Como resultados tivemos quatro aplicativos em fase de protótipos funcionais e um em fase de ideia e concepção.

Por fim, a etapa de premiação quando todos os participantes foram envolvidos. Após a deliberação da comissão julgadora que contou com a presença de representantes da empresa anfitriã do evento, a equipe vencedora foi premiada. Os vencedores receberam um micro-estágio na empresa sede do evento, que será realizado de forma complementar as atividades acadêmicas. Os alunos poderão atuar em atividades relacionadas aos seus interesses acadêmicos (front-end, back-end, design ou projetos), relacionando ou não aos trabalhos conduzidos na Universidade. Os demais envolvidos, incluindo a organização do evento e representantes dos patrocinadores, receberam medalhas de participação conforme pode ser visto na foto da Fig.5.

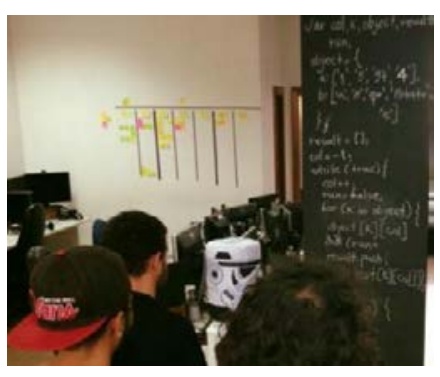

(a)

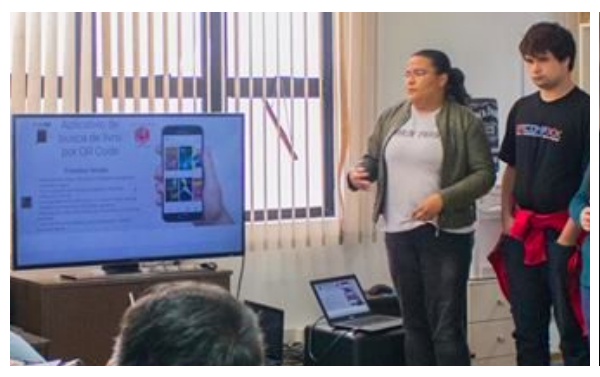

(b)

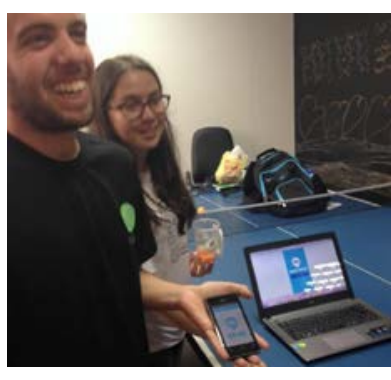

(c)

Figura 4. Desafio de programação realizado (a), apresentação dos produtos, em (b) pitches de 5 minutos e em (c) demonstrações. 


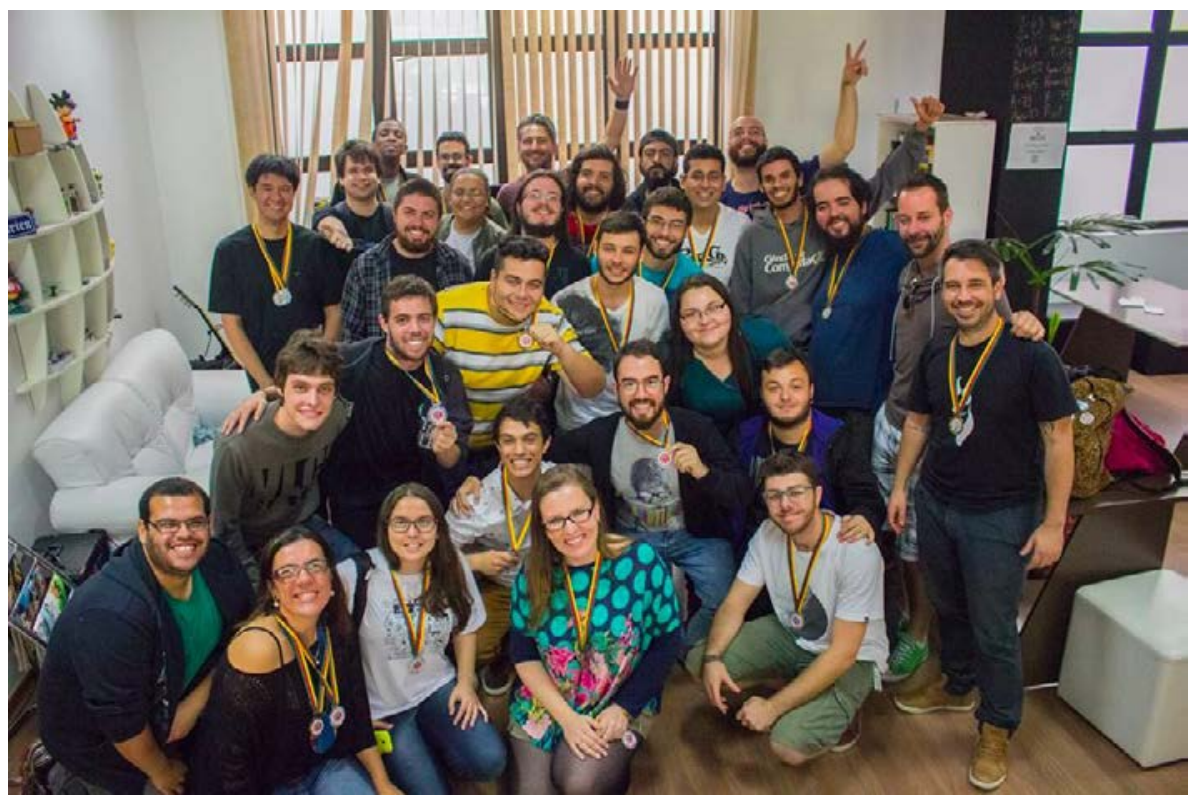

Figura 5. Premiação.

\section{Resultados Pós-Evento}

Como etapa pós-evento foi lançado um questionário de participação voluntária para que pudéssemos avaliar a recepção do evento pela perspectiva dos participantes. Questões a respeito da apreciação geral, organização e comida/bebida obtiveram $100 \%$ de respostas "excelente". A avaliação também possibilitou analisar a premissa deste artigo: hackathons como ferramentas de estímulo a aprendizagem. As figuras Fig.6 (a) e (b) destacam o estágio do curso e a opinião dos participantes quanto ao objetivo e formato do evento, respectivamente. Observamos que a maioria dos participantes está no meio do curso e todos classificaram como Bom $(12,5 \%)$ e Excelente $(87,5 \%)$ o formato do evento. Fortalecendo essa tendência dos dados obtidos, destacamos o seguinte depoimento de um dos participantes: "Aprendi muito nesse evento graças aos colegas e professores. Desde o básico a coisas avançadas do design! Também vi o que funciona ou não na hora da programação. Essas 36 horas me ensinaram coisas que eu demoraria muito mais tempo pra aprender. E achei maravilhoso ter medalha de prata do último ao segundo lugar. Não tenho nada de ruim pra falar sobre o evento, me diverti e aprendi muito! Só estou esperando o próximo evento :)".

Também chamou atenção nos resultados obtidos neste questionário a impressão dos participantes do local e da duração do evento. Quanto ao local a grande maioria classificou como Excelente (87,5\%) e 12,5\% como Bom (vide Fig.6 (d)). A imersão em um ambiente empresarial foi muito importante como ferramenta de motivação. Já a avaliação do tempo de duração do evento demonstrou que a maioria aprovou as 36 horas $(87,5 \%)$ conforme Fig.6 (c).

Além disso, percebeu-se como resultado do evento o interesse dos acadêmicos na aplicação dessa experiência de integração entre a Computação e o Design em períodos maiores e presente no calendário das instituições. Dessa forma, conseguimos ofertar aos alunos de Engenharia da Computação, Ciências da Computação, Design Gráfico e Design Digital da UFPel uma disciplina em conjunto, com 32 vagas divididas

Proceedings of the WEI'2016 (Julho 2016) 
entre os 4 cursos, que ocorrerá durante o primeiro semestre de 2016. Com essa iniciativa, as experiências proporcionadas pelo evento poderão ser contempladas por alunos matriculados nos respectivos cursos e que não tiveram, por algum motivo, a oportunidade em participar do evento. A metodologia da disciplina seguirá os mesmos padrões do CodeArena, exceto pelo tempo de desenvolvimento, e será ministrada por dois dos professores organizadores do evento.

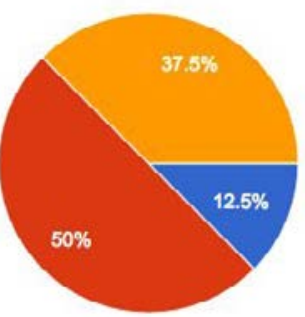

(a)

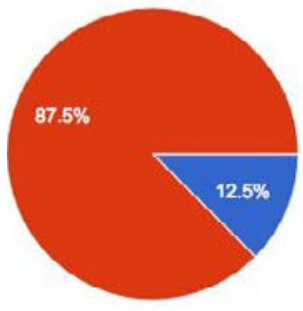

(c)

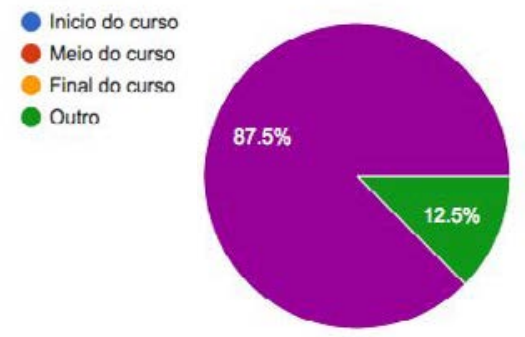

(b)
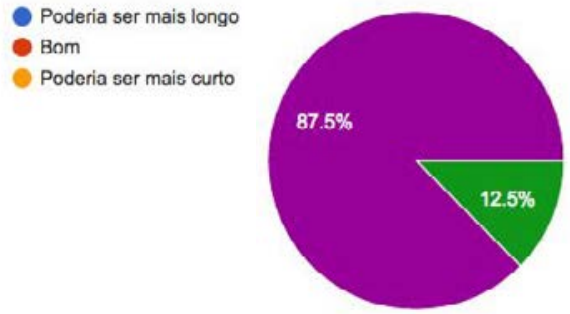

(d)
1 - Muito insatisfatórin

2 - Insatisfatório

3 - Médin

4-Bom

5 - Excelente

Figura 6. Algumas respostas do formulário de avaliação do evento.

\section{Conclusões e Trabalhos Futuros}

Além dos resultados tecnológicos e de caráter empreendedor, a realização do evento trouxe ganhos intangíveis para os envolvidos. Os ganhos serão descritos nos próximos parágrafos sob as perspectivas da universidade e das empresas envolvidas, relatando as vantagens percebidas pelos envolvidos de ambas perspectivas.

Da perspectiva da universidade, foram inúmeros os aspectos observados como vantajosos na realização de um evento deste tipo, segundo opiniões coletadas em conversas informais com os professores e alunos que participaram do evento. Do ponto de vista dos alunos, tivemos os próprios integrantes dos times, que participaram ativamente na concepção dos protótipos resultantes. Os participantes gostaram do formato do evento e do desafio de desenvolverem, em curto espaço de tempo, projetos aplicados para resolver algum problema real da sociedade. Relataram que tais desafios não são comuns nos trabalhos acadêmicos e que os conhecimentos necessários para desenvolvê-los vem para complementar os ensinamentos teóricos vividos em sala de aula. Estes mesmos alunos, relataram que ficaram muito empolgados por estarem dentro do ambiente da empresa. Segundo eles, o ambiente proporcionou uma sensação de Proceedings of the WEI'2016 (Julho 2016) 
motivação e vivência de um dia como um profissional. Aliado ao desafio dado pelo curto prazo do evento, que simulava uma demanda real do mercado de trabalho, eles se sentiram como se tivessem uma entrega de uma release do software para avaliação do cliente.

Outro fator importante, citado pelos participantes, foi a presença de mentores. Segundo eles, os mentores tiraram dúvidas e auxiliaram em questões práticas que permitiram aos times ter mais agilidade e qualidade na entrega dos protótipos. Como mentores, participaram alunos, integrantes da empresa Júnior da UFPel (Hut8), profissionais das empresas hospedeira e patrocinadoras, e professores da UFPel e IFSul, dos cursos de Computação e Design. Os mentores relataram que a experiência proporcionou a aproximação com os alunos e a troca de experiências além de apoiar os participantes com dicas de programação, design e apoio técnico nas ferramentas e técnicas utilizadas pelos times.

Para os mentores, em especial os professores, o evento proporcionou conhecer melhor as habilidades de alguns alunos, como por exemplo identificar talentos em frameworks, linguagens de programação e tecnologias. $\mathrm{O}$ evento proporcionou mapear competências para futuras bolsas ou projetos de seus laboratórios. Além é claro da integração entre alunos e professores. Os alunos da empresa júnior também aproveitaram o momento para conhecer novos talentos e fazer networking com profissionais da empresa que estavam no evento.

Essa integração foi auxiliada por mentores com vivência e domínio desde a parte de negócios, passando pela experiência de usuário e também com pleno domínio em desenvolvimento. Ainda para tentar dar maior similaridade ao dia-a-dia do mercado de trabalho, além de afastar um pouco do ambiente acadêmico com o qual os alunos estão acostumados e confortáveis, toda essa experiência aconteceu dentro de uma empresa alemã que possui uma de suas filiais na cidade de Pelotas. Também foi estimulado o empreendedorismo com foco na resolução de problemas locais e até mesmo outros mais amplos que com a globalização ultrapassam barreiras territoriais e atingem uma parte significativa da sociedade.

Sob a perspectiva da empresa sede do evento, as vantagens foram inúmeras. Além de permitir que alunos e professores conhecessem suas instalações, o empresário e sua equipe puderam se aproximar dos alunos e professores de forma a mapear talentos para futura captação para seus projetos. Como a empresa sede foi uma empresa de design e marketing digital, eles puderam sugerir aos alunos tanto de computação quanto de design, algumas técnicas e ferramentas utilizadas na empresa, através dos mentores disponibilizados por eles. Os patrocinadores, puderam enviar mentores e participar de todo o evento. Nesta edição, nenhum dos patrocinadores, exceto a empresa sede, pôde enviar mentores para participar na data do evento, portanto não foi possível coletar a percepção destas empresas com relação aos ganhos com a mentoria no evento. Mas em conversas com os empresários, foi possível perceber, que de posse dos dados sobre os participantes, eles farão aproximações com o intuito de identificação de talentos, além de aproximação entre as próprias empresas envolvidas, visando futuros acordos comerciais. Ainda na perspectiva da universidade, uma vantagem importante foi a aproximação entre os cursos de design e computação. Tal demanda, inicialmente identificada pelos alunos de design e computação, através de seus diretórios 
acadêmicos, foi a grande motivadora da criação deste evento com esse formato integrador. A aproximação dos dois cursos permitiu que os grupos fossem formados de forma interdisciplinar, similar a formação dos times nas empresas. Tal interdisciplinaridade, trouxe a complementaridade na condução dos projetos de desenvolvimento dos aplicativos. Enquanto uns programavam front e backend, o designer atuava na concepção do layout e telas da aplicação, inclusive, em alguns times, desenhando telas que não seriam implementadas a tempo do evento, mas que poderiam contribuir para a apresentação de funcionalidades de futuras releases. A integração dos times proporcionou uma troca, em tempo real, de demandas de mudanças que impactaram tanto o design quanto a implementação de front e backend, proporcionando que os integrantes dos times tivessem que agir de forma rápida às mudanças. $\mathrm{O}$ uso de técnicas de gerenciamento ágil de projetos, proporcionou aos grupos que aplicaram, um rápido planejamento e visualização das atividades necessárias e o andamento do desenvolvimento. O grupo responsável pelo desenvolvimento do projeto ClassFeed usou ciclos rápidos de desenvolvimento e teste do aplicativo, através da aplicação de Scrum. A implementação do Kanban aliado às cores, segundo eles, trouxe agilidade e garantiu a produtividade do grupo no tempo previsto do evento.

Como trabalhos futuros, já estamos trabalhando na concepção do Code Arena de 2016. Trabalharemos com uma metodologia similar, aplicando as lições aprendidas e melhorando a interação com todos os envolvidos.

\section{Referências}

Heikki Topi and Allen Tucker. Computing Handbook, Third Edition: Information

Systems and Information Technology. CRC Press, 2014.

Briscoe, Gerard, and Catherine Mulligan. "Digital innovation: The hackathon phenomenon." London: Creativeworks London Work Paper 6 (2014). (Disponível em:

http://www.creativeworkslondon.org.uk/wp-content/uploads/2013/11/Digital-Innovat ion-The-Hackathon-Phenomenon1.pdf )

Fassbinder, Aracele Garcia de O., Lílian Cristina de Paula, e João Cláudio D. Araújo. "Experiências no estímulo à prática de Programação através do desenvolvimento de atividades extracurriculares relacionadas com as competições de conhecimentos". In: Anais do Workshop sobre Educação em Computação (WEI), Congresso da Sociedade Brasileira de Computação (CSBC). 2012.

de Barros, Louise G., and Janne Yukiko Y. Oeiras. "Extensão universitária em Computação por meio da Olimpíada Brasileira de Informática." In: Anais do Workshop sobre Educação em Computação (WEI), Congresso da Sociedade Brasileira de Computação (CSBC). (2010) Disponível em: http://www.lbd.dcc.ufmg.br/colecoes/wei/2010/009.pdf

Arnab Nandi and Meris Mandernach. 2016. Hackathons as an Informal Learning Platform. In Proceedings of the 47th ACM Technical Symposium on Computing Science Education (SIGCSE '16). ACM, New York, NY, USA, 346-351. DOI=http://dx.doi.org/10.1145/2839509.2844590

Proceedings of the WEI'2016 (Julho 2016) 